\title{
Aldosterone-induced cardiac damage in primary aldosteronism depends on its subtypes
}

\author{
Satoshi Higuchi ${ }^{1}$, Hideki Ota ${ }^{1,2}$, Yuta Tezuka ${ }^{3,4}$, Kazumasa Seiji ${ }^{1}$, Hidenobu Takagi ${ }^{1,5}$, Jongmin Lee ${ }^{6}$, Yi-Wei Lee ${ }^{7}$, \\ Kei Omata ${ }^{3,4}$, Yoshikiyo Ono ${ }^{3,4}$, Ryo Morimoto ${ }^{3}$, Masataka Kudo ${ }^{3}$, Fumitoshi Satoh ${ }^{3,4}$ and Kei Takase ${ }^{1}$ \\ 'Department of Diagnostic Radiology, Tohoku University Hospital, Sendai, Miyagi, Japan \\ 2Department of Advanced MRI Collaboration Research, Tohoku University Graduate School of Medicine, Sendai, Miyagi, Japan \\ ${ }^{3}$ Division of Nephrology, Endocrinology and Vascular Medicine, Department of Medicine, Tohoku University Hospital, Sendai, Miyagi, Japan \\ ${ }^{4}$ Division of Clinical Hypertension, Endocrinology and Metabolism, Tohoku University Graduate School of Medicine, Sendai, Miyagi, Japan \\ ${ }^{5}$ Department of Radiology, The University of British Columbia, Vancouver, Canada \\ ${ }^{6}$ Department of Radiology, School of Medicine, Kyungpook National University, Daegu, Korea \\ ${ }^{7}$ Department of Radiology, Kaohsiung Chang Gung Memorial Hospital and Chang Gung University College of Medicine, Kaohsiung, Taiwan \\ Correspondence should be addressed to H Ota: h-ota@rad.med.tohoku.ac.jp
}

\begin{abstract}
Objectives: This study compared cardiac function, morphology, and tissue characteristics between two common subtypes of primary aldosteronism (PA) using a 3T MR scanner. Design: A retrospective, single-center, observational study.

Methods: We retrospectively reviewed 143 consecutive patients with PA, who underwent both adrenal venous sampling and cardiac magnetic resonance. We acquired cine, late gadolinium enhancement, and pre- and postcontrast myocardial T1-mapping images. Results: PA was diagnosed as unilateral aldosterone-producing adenoma (APA) in 70 patients and bilateral hyperaldosteronism (BHA) in 73. The APA group showed significantly higher plasma aldosterone concentration (PAC) and aldosterone to renin rate (ARR) than the BHA group. After controlling for age, sex, antihypertensive drugs, systolic and diastolic blood pressure, and disease duration, the parameters independently associated with APA were: left ventricular end-diastolic volume index (EDVI: adjusted odds ratio $(\mathrm{aOR})=1.06(95 \% \mathrm{Cl}: 1.030-1.096), P<0.01)$, end-systolic volume index (ESVI: $1.06(1.017-1.113), P<0.01)$, stroke index (SI: $1.07(1.020-1.121)$, $P<0.01)$, cardiac index (Cl: 1.001 (1.000-1.001), $P<0.01)$, and native T1 (1.01 (1.000-1.019), $P=0.038)$. Weak positive correlations were found between PAC and EDVI $(R=0.28$, $P<0.01)$, ESVI $(0.26, P<0.01)$, and SI $(0.18, P=0.03)$; and between ARR and EDVI $(0.25$, $P<0.01)$, ESVI $(0.24, P<0.01)$, and native T1 $(0.17, P=0.047)$. Conclusions: APA is associated with greater LV volumetric parameters and higher native T1 values, suggesting a higher risk of volume overload and myocardial damage.
\end{abstract}

\section{Key Words}

- aldosterone-induced cardiac damage

- aldosterone-producing adenoma

- bilateral hyperaldosteronism

- cardiac magnetic resonance

\section{Introduction}

Primary aldosteronism (PA) is one of the most frequent forms of secondary hypertension with an estimated prevalence of approximately $11 \%$ in hypertensive populations $(1,2)$. Some patients with PA suffer from refractory hypertension due to excessive and autonomous aldosterone production.
Independent of its effect on blood pressure, such excess in aldosterone has been associated with cardiovascular damage (3), including myocardial inflammation (4), excessive collagen deposition and fibrosis in myocardial tissue $(5,6)$, vascular remodeling, arterial stiffening, and endothelial dysfunction (7). In aldosterone-induced

Endocrine Connections (2021) 10, 29-36 
cardiac fibrosis, it causes extracellular matrix deposition by enhancing the transcription of the collagen type I and III genes via mineralocorticoid receptor or angiotensin II receptors in the myocardium (8). Patients with PA suffer from cardiovascular events such as coronary artery disease, atrial fibrillation, and heart failure more than those with essential hypertension (EH) and matched cardiovascular risk profile, resulting in increased cardiovascular mortality $(9,10)$.

The two most common causes of PA are unilateral aldosterone-producing adenomas (APAs) and bilateral hyperaldosteronism (BHA) (1, 2). Distinguishing APAs from BHA in patients with $\mathrm{PA}$ is important because the APAs can be treated by unilateral or partial adrenalectomy. APAs often secrete higher amounts of corticosterone, deoxycorticosterone, and the hybrid steroids, 18-hydroxycortisol and 18-oxocortisol, compared with the adrenals of BHA patients $(11,12)$. In echocardiographic assessments, the left atrial volume, left ventricular end-diastolic and end-systolic diameters, left ventricular mass (LVM), and prevalence of left ventricular hypertrophy (LVH) were found to be greater in patients with APA than in those with BHA (13). However, there have been few reports about the differences in myocardial tissue characteristics among subtypes of PA.

Cardiac magnetic resonance (CMR) is a valuable tool for the non-invasive, highly accurate, and reproducible assessment of cardiac morphology and function, and for myocardial tissue characterization. Cine MR images allow the evaluation of left ventricular morphology and volumetric parameters. Late gadolinium enhancement (LGE) can be used to detect focal myocardial fibrosis using gadolinium-based contrast agents. Moreover, myocardial T1 mapping provides native T1 values reflecting myocardial tissue properties such as edema or fibrosis, and extracellular volume (ECV) indicating the extent of extracellular space, which is increased due to excessive collagen deposition and fibrosis (14). This technique is a sensitive tool to detect diffuse myocardial pathophysiological changes (15).

Past CMR studies about PA demonstrated a significant increase in the frequency of cardiac volume overload, left ventricle hypertrophy, and non-infarct LGE compared with essential hypertension $(16,17)$. Another study compared ECV between patients with PA and healthy subjects, using T1 mapping, and demonstrated no significant differences between the two groups; however, the study sample size was small, and the difference among subtypes of PA was not taken into consideration (18). Among PA patients, the ones with APA, with higher hormonal activity than those with BHA, should also have higher cardiac risk, even in the absence of clinical symptoms. The demonstration of subclinical cardiac risk in APA patients by CMR could support the clinical importance of adrenal venous sampling (AVS) and surgical intervention in PA patients.

The purpose of this study was to compare myocardial morphology and function, and tissue characteristics, between patients with APA and BHA who had no history of cardiac disease using a 3T MR scanner.

\section{Methods}

\section{Ethics}

This retrospective study was approved by the Ethics Committee of the Tohoku University School of Medicine (approval \#2019-1-142), who waived the requirement of written informed consent.

\section{Patients}

One hundred fifty-seven consecutive patients with newly diagnosed PA, who underwent both AVS and CMR, were included in this study. All patients were diagnosed by endocrinologists from September 2015 to May 2018 with the captopril challenge test and/or saline infusion test, in addition to plasma aldosterone concentration (PAC), aldosterone to renin ratio (ARR), and plasma renin activity, in accordance with the current Japanese guidelines (19).

Exclusion criteria were previous history of cardiac events (heart failure, ischemic heart disease, valvular disease, cardiomyopathy, congenital heart disease, and arrhythmia), and severe renal dysfunction (estimated glomerular filtration rate $<30 \mathrm{~mL} / \mathrm{min}$ ) (Fig. 1). Patient demographic characteristics were collected, including sex, age, BMI, family history of $\mathrm{EH}$, medical history of diabetes mellitus, smoking history, number of antihypertensive drugs (counted at the first visit and at AVS), systolic and diastolic blood pressure (measured at the first visit and at AVS), pulse rate (measured at the first visit and at AVS), and disease duration (the interval from the appearance of subjective symptoms or detection of hypertension to diagnosis). Blood pressure was measured three times with an appropriate-sized cuff (medium or large) in the sitting position after a rest period of greater than 5 min using an automated blood pressure measuring device (WatchBP Office; Microlife Corporation, Taipei, Taiwan); the average value of recorded measurement was considered for the present study. Antihypertensive drugs counted at first visit

This work is licensed under a Creative Commons Attribution-NonCommercial-NoDerivatives 4.0 elfternationab ficense.ifica.com at 04/26/2023 07:24:31AM 


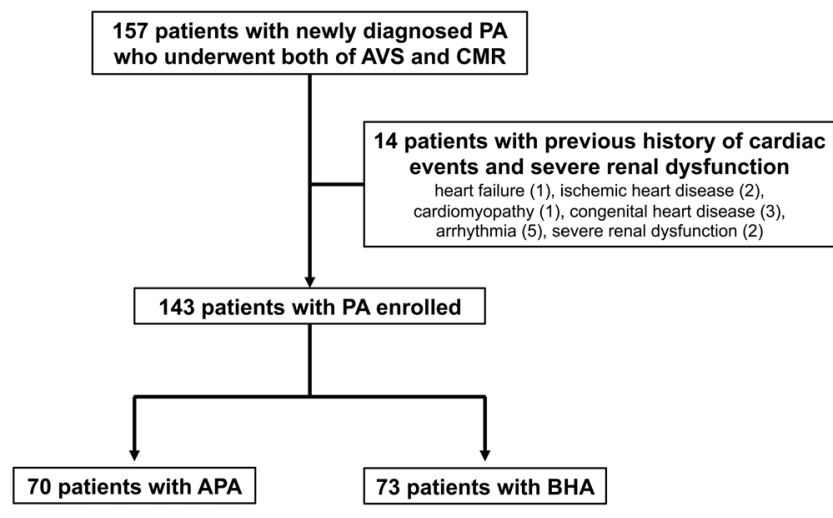

\section{Figure 1}

Flow diagram and patient disposition. One hundred fifty-seven consecutive patients newly diagnosed with primary aldosteronism (PA), who underwent both adrenal venous sampling (AVS) and cardiac magnetic resonance (CMR), were included. Fourteen patients were excluded due to previous history of cardiac events (heart failure, ischemic heart disease, cardiomyopathy, congenital heart disease, or arrhythmia) or severe renal dysfunction (estimated glomerular filtration rate $<30 \mathrm{~mL} / \mathrm{min}$ ). Among the 143 patients analyzed, the subtypes of PA were diagnosed by AVS as unilateral aldosterone-producing adenoma (APA) in 70 patients and bilateral hyperaldosteronism (BHA) in 73 .

included calcium channel blockers (CCB), angiotensinconverting enzyme inhibitors (ACEIs), angiotensin receptor blockers (ARBs), alpha-blockers, beta-blockers, diuretics, and aldosterone antagonists (Supplementary Table 2, see section on supplementary materials given at the end of this article). Blood pressure at AVS was measured free of aldosterone antagonists including spironolactone (Supplementary Table 3).

A fixed-dose combination incorporating two antihypertensive drugs into one pill was counted as two drugs. Blood examination data were also collected, including plasma aldosterone concentration (PAC), aldosterone/renin ratio (ARR), and brain natriuretic peptide (BNP).

\section{CMR protocol}

All patients were imaged with a 3T whole-body MR scanner (MAGNETOM Trio A Tim System; Siemens Healthineers) at around the same time of the diagnosis of PA. Scan protocols included cine, precontrast and postcontrast T1 map, and late gadolinium enhancement (LGE). Cine images were acquired at 20 frames per slice per beat in the short axis, long axis, and four-chamber views. LGE and postcontrast T1 map were acquired 10-15 min after the administration of $0.1 \mathrm{mmol} / \mathrm{kg}$ body weight of gadolinium-contrast agent (gadobutol, Gadovist; Bayer, Osaka, Japan). We obtained LGE images in the short axis, long axis and four-chamber view, and three-slice T1 maps in the short axis view. T1 maps were acquired by using a modified Look-Locker inversion recovery (MOLLI) technique. To accurately measure longer native $\mathrm{T} 1$ times independently of heart rate, we applied the (8(2)2) scheme indicating two inversion pulses with the acquisition of images for eight heartbeats, followed by a recovery of two heartbeats, and a second inversion pulse with images acquired for two heartbeats. Postcontrast T1 mapping was acquired using a 3(3)3(3)5 scheme, targeting a shortened T1 time. In-plane motion correction was performed and T1 maps were automatically generated. Details of the cine, LGE, and T1 mapping parameters are provided in Supplementary Table 1.

\section{Image analysis}

We analyzed MR images using a workstation (Ziostation2; Ziosoft, Tokyo, Japan). The observers were blinded to patient characteristics. We measured end-systolic and enddiastolic ventricle septum wall thickness, end-diastolic, end-systolic, and stroke volume index (EDVI, ESVI, and SI, respectively) and cardiac index (CI) from short-axis cine images. The left ventricular myocardial mass index (LVMI) was also acquired with short-axis cine images using the Simpson technique. An expert cardioradiologist evaluated the presence of LGE. LGE was defined as the signal intensity of greater than two times S.D. above the average of normal myocardium (20).

Two radiologists independently measured native T1 values, so as to analyze interobserver reproducibility. One of the two radiologists measured pre- and postcontrast myocardial T1 and blood T1 values twice, with an interval greater than 3 months, to analyze intraobserver reproducibility. Regions of interests for the evaluation of native $\mathrm{T} 1$ and postcontrast myocardial $\mathrm{T} 1$ were placed on the mid-myocardial layers of the basal septum (Fig. 2). T1 values measured by one reviewer were taken to be representative of individual segments. ECVs were calculated using the following formula: (1 - hematocrit) $\times\left(1 / \mathrm{T} 1_{\text {myo post }}-1 / \mathrm{T} 1_{\text {myo pre }}\right) /\left(1 / \mathrm{T} 1_{\text {blood post }}-1 / \mathrm{T} 1_{\text {blood pre }}\right)$.

\section{Adrenal venous sampling for subtype diagnosis}

All patients underwent AVS to differentiate between APA and BHA 2 or 3 days after CMR. We performed AVS with cosyntropin (ACTH) stimulation to maximize the gradient in cortisol concentration from the adrenal veins to the IVC and confirm the successful sampling from the adrenal veins (21). To correct non-uniform dilution effects between the right and left adrenal veins, we calculated

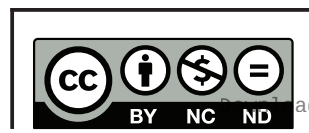

This work is licensed under a Creative Commons Attribution-NonCommercial-NoDerivatives 4.0 Internationad dicense ifica. com at 04/26/2023 07:24:31AM 


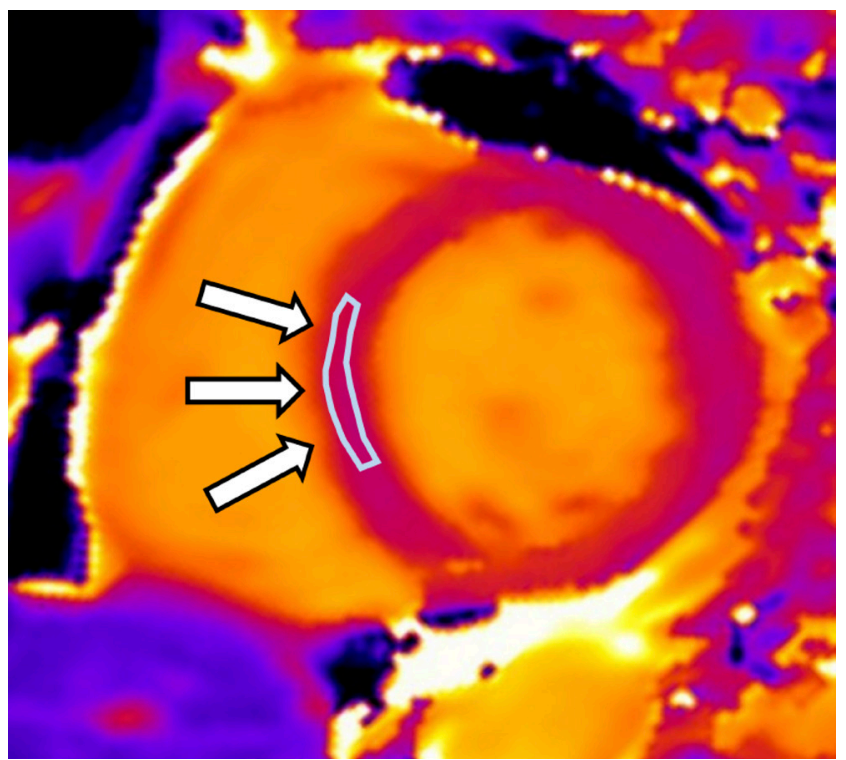

\section{Figure 2}

Region of interest for the evaluation of myocardial T1 value. Image of native T1 map from a 49-year-old man with aldosterone-producing adenoma. A sample region of interest (arrow and circle) for the evaluation of myocardial $\mathrm{T} 1$ values is shown on the mid-myocardial layers of the basal septum.

the lateralization index (LI), the ratio of the higher to the lower aldosterone/cortisol (A/C) ratio (22). Unilateral APAs were diagnosed when the LI after cosyntropin stimulation was 2.6 or greater $(23)$.

\section{Statistical analysis}

Descriptive statistics are presented as mean \pm S.D., for continuous variables and as number of cases and percentages per group for categorical variables. Nonnormally distributed variables are shown as median with interquartile range (IQR). The normality of MR parameters and demographic data were tested using the KolmogorovSmirnov test $(P>0.05)$. Variables were compared between the APA and BHA groups using Student's t-tests for normal variables or Mann-Whitney's U tests for non-normal ones. For dichotomous data, the Fisher's exact test was used to analyze differences in proportions between the groups. Multivariable logistic regression models were used to analyze the association between MR parameters and the APA group (the BHA group being defined as the reference group) adjusting for age, sex, number of antihypertensive drugs, systolic and diastolic blood pressure, and disease duration considered as confounders. The Pearson correlation coefficient was used to evaluate correlations between parameters. Intra- and interobserver reproducibility of pre- and postcontrast myocardial T1 values were evaluated using the intraclass correlation coefficients (ICC).
Based on the $95 \%$ CI of the ICC, values less than 0.5, 0.5$0.75,0.75-0.9$, and greater than 0.9 are indicative of poor, moderate, good, and excellent reliability, respectively. Statistical analyses were performed using SPSS, version 21.0 (IBM). $P$-values $<0.05$ were considered statistically significant.

\section{Results}

\section{Patient characteristics}

Among the 157 patients with PA, 143 were included in the analysis, as 14 had a history of cardiovascular diseases or severe renal dysfunction: heart failure $(n=1)$, ischemic heart disease $(n=2)$, cardiomyopathy $(n=1)$, congenital heart disease $(n=3)$, arrhythmia $(n=5)$, and severe renal dysfunction $(n=2)$.

Subtypes of PA were diagnosed by AVS as unilateral APA in 70 (male, 45) and BHA in 73 (male, 30) patients. Among the 70 patients with APA, 59 underwent unilateral or partial adrenalectomy and the diagnosis was confirmed pathologically, 6 underwent CT-guided radiofrequency ablation, and 5 were waiting for or unwilling to undergo surgical intervention. The baseline patient characteristics are summarized in Table 1.

In demographic data, the male proportion (APA vs BHA $=64 \%$ vs $41 \%, P<0.01$ ), disease duration (8.5 (IQR, $4-15)$ vs $5(2-10.5)$ years, $P=0.03)$, and the number of antihypertensive drugs ( $(\mathrm{IQR}, 1-3)$ vs $1(1-2), P<0.01$ ) were significantly larger in the APA than in the BHA group. There were no significant differences between the two groups in the other demographic parameters.

\section{Hormonal data and brain natriuretic peptide (BNP)}

The APA group showed significantly higher PAC levels and ARR than the BHA group (PAC: 41.3 (IQR: 29.0-61.7) vs 22.7 (17.8-27.2) ng/dL, $P<0.01$; ARR: 152.3 (76.0-298) vs 63.0 (40.5-92.6) $\mathrm{ng} / \mathrm{dL}$ per $\mathrm{ng} /(\mathrm{mL} \mathrm{h}), P<0.01)$. BNP demonstrated no significant difference between the groups (Table 1).

\section{LV volumetric parameters and tissue characteristics}

The left ventricular (LV) end-systolic walls were significantly thicker in the APA than in the BHA group $(16.3 \pm 2.4$ vs $15.4 \pm 2.1 \mathrm{~mm}, P=0.02)$. The APA group also showed significantly higher left ventricular EDVI (76.9 (IQR 69.7-85.7) vs $67.0(59.4-77.9) \mathrm{mL} / \mathrm{m}^{2}, P<0.01$, 
Table 1 Patient demographic characteristics.

\begin{tabular}{|c|c|c|c|}
\hline & $\begin{array}{c}\text { APA group } \\
(n=70)\end{array}$ & $\begin{array}{l}\text { BHA group } \\
\quad(n=73)\end{array}$ & $P$ value \\
\hline Age (years) & $52 \pm 11$ & $52 \pm 12$ & 0.76 \\
\hline Male & $45(64 \%)$ & $30(41 \%)$ & $<0.01^{a}$ \\
\hline BMI (kg/m²) & $24.6 \pm 4.0$ & $25.3 \pm 4.2$ & 0.29 \\
\hline $\begin{array}{l}\text { Family history of } \\
\text { hypertension }\end{array}$ & $50(71 \%)$ & $57(79 \%)$ & 0.33 \\
\hline DM & $10(14 \%)$ & $8(11 \%)$ & 0.62 \\
\hline Smoking history & $35(50 \%)$ & $42(58 \%)$ & 0.4 \\
\hline $\begin{array}{l}\text { Number of } \\
\text { antihypertensive drugs }{ }^{a}\end{array}$ & $2(1-3)$ & $1(1-2)$ & $<0.01^{a}$ \\
\hline $\begin{array}{l}\text { Systolic BP }(\mathrm{mmHg}) \\
\text { at first visit }\end{array}$ & $148 \pm 16$ & $148 \pm 21$ & 0.89 \\
\hline $\begin{array}{l}\text { Diastolic BP }(\mathrm{mmHg}) \\
\text { at first visit }\end{array}$ & $96 \pm 10$ & $97 \pm 14$ & 0.73 \\
\hline $\begin{array}{l}\text { Systolic BP (mmHg) } \\
\text { at AVS }\end{array}$ & $138 \pm 17$ & $138 \pm 16$ & 0.45 \\
\hline $\begin{array}{l}\text { Diastolic BP }(\mathrm{mmHg}) \\
\text { at AVS }\end{array}$ & $90 \pm 11$ & $89 \pm 11$ & 0.39 \\
\hline $\begin{array}{l}\text { Pulse rate (b.p.m.) } \\
\text { at first visit }\end{array}$ & $74(67-83)$ & $74(70-85)$ & 0.45 \\
\hline Pulse rate (b.p.m.) at AVS & $76(68-85)$ & $81(70-90)$ & 0.08 \\
\hline Disease duration (years) ${ }^{a}$ & $8.5(4-15)$ & $5(2-11)$ & $<0.01^{a}$ \\
\hline PAC (ng/dL) ${ }^{a}$ & $41.3(29.0-61.7)$ & $22.7(17.8-27.2)$ & $<0.01^{a}$ \\
\hline $\mathrm{ARR}^{\mathrm{a}}$ & $152.3(76-298)$ & 63 (40.5-92.6) & $<0.01^{a}$ \\
\hline BNP (pg/mL) & $9.4(0-16.5)$ & $6.3(0-13.4)$ & 0.13 \\
\hline $\mathrm{Ht}(\%)$ & $40.9 \pm 0.5$ & $40 \pm 0.5$ & 0.21 \\
\hline
\end{tabular}

Values are $n(\%)$, mean \pm S.D., or median (interquartile range).

alndicates statistical significance.

APA, aldosterone-producing adenoma; ARR, aldosterone to renin ratio; AVS, adrenal venous sampling; BHA, bilateral hyperaldosteronism; BNP brain natriuretic peptide; $\mathrm{BP}$, blood pressure; $\mathrm{DM}$, diabetes mellitus; $\mathrm{Ht}$, hematocrit; PAC, plasma aldosterone concentration.

ESVI (32.8 (25.7-38.2) vs $25.9 \quad(19.0-32.3) \quad \mathrm{mL} / \mathrm{m}^{2}$, $P<0.01)$, SI $\left(46.4 \pm 8.6\right.$ vs $\left.41.5 \pm 8.4 \mathrm{~mL} / \mathrm{m}^{2}, P<0.01\right)$, CI $\left(3140 \pm 637\right.$ vs $\left.2856 \pm 593 \mathrm{~mL} / \mathrm{min} / \mathrm{m}^{2}, P<0.01\right)$, MI $\left(55.4 \pm 15\right.$ vs $\left.49.3 \pm 12.2 \mathrm{~g} / \mathrm{m}^{2}, P=0.02\right)$. Other MR parameters (end-diastolic wall thickness, LVEF, Native T1, and ECV) demonstrated no significant differences between the two groups in the univariate analysis (Table 2).

Seven patients with APA and four with BHA did not undergo postcontrast scan due to mild to moderate renal dysfunction (eGFR; 30-45 mL/min/1.73 $\mathrm{m}^{2}$ ) or allergy to contrast medium. The presence of LGE demonstrated no significant difference between APA and BHA (16\% $(10 / 63)$ vs $20 \%(14 / 69), P=0.65)$. After controlling for age, sex, number of antihypertensive drugs, systolic and diastolic blood pressure, and disease duration, the following parameters were independently associated with the presence of APA, the BHA group being taken as the reference group (Fig. 3): EDVI (adjusted odds ratio $(\mathrm{aOR})=1.06$ (95\% CI: 1.030-1.096), $P<0.01)$, ESVI $(\mathrm{aOR}$ $=1.06$ (1.017-1.113), $P<0.01)$, SI (1.07 (1.020-1.121), $P<0.01)$, CI (1.001 (1.000-1.001), $P<0.01)$, and native T1 (1.01 (1.000-1.019), $P=0.038)$.
Table 2 MR parameters between APA and BHA.

\begin{tabular}{l}
\hline \\
LV EDWT $(\mathrm{mm})$ \\
LV ESWT $(\mathrm{mm})^{a}$ \\
LV EF $(\%)$ \\
LV EDVI $\left(\mathrm{mL} / \mathrm{m}^{2}\right)^{a}$ \\
LV ESVI $\left(\mathrm{mL} / \mathrm{m}^{2}\right)^{a}$ \\
LV SI $\left(\mathrm{mL} / \mathrm{m}^{2}\right)^{a}$ \\
LV CI $\left(\mathrm{mL} / \mathrm{min}^{2} \mathrm{~m}^{2}\right)^{a}$ \\
LV MI $\left(\mathrm{g} / \mathrm{m}^{2}\right)^{\mathrm{a}}$ \\
Native T1 $(\mathrm{ms})$ \\
ECV $(\%)$
\end{tabular}

\begin{tabular}{c}
\hline APA group \\
\hline $12.2 \pm 2.0$ \\
$16.3 \pm 2.4$ \\
$58.9 \pm 7.1$ \\
$76.9(69.7-85.7)$ \\
$32.8(25.7-38.2)$ \\
$46.4 \pm 8.6$ \\
$3140 \pm 637$ \\
$55.4 \pm 15$ \\
$1255 \pm 45$ \\
$29.2 \pm 3.3$ \\
\hline
\end{tabular}

\begin{tabular}{cccc}
\hline BHA group & & P value \\
\cline { 1 - 1 } $11.8 \pm 2.1$ & & 0.21 \\
$15.4 \pm 2.1$ & & $0.02^{a}$ \\
$61.1 \pm 8.5$ & & 0.42 \\
$67.0(59.4-77.9)$ & & $<0.01^{a}$ \\
$25.9(19.0-32.3)$ & & $<0.01^{a}$ \\
$41.5 \pm 8.4$ & & $<0.01^{a}$ \\
$2856 \pm 593$ & & $<0.01^{a}$ \\
$49.3 \pm 12.2$ & & $0.02^{a}$ \\
$1253 \pm 49$ & & 0.73 \\
$29.8 \pm 3.5$ & & 0.44
\end{tabular}

Indicates statistical significance.

APA, aldosterone-producing adenoma; $\mathrm{BHA}$, bilateral hyperaldosteronism; $\mathrm{Cl}$, cardiac index; ECV, extra cellular volume; EF, ejection fraction; EDVI, end-diastolic volume index; EDWT, end-diastolic wall thickness; ESVI, end-systolic volume index; ESWT, end-systolic wall thickness; LV, left ventricle; MI, mass index; SI, systolic volume index.

\section{Correlation between MR parameters and logarithmic PAC and ARR}

We performed a logarithmic transformation to obtain a normal distribution of PAC and ARR. The logarithmic PAC showed a weak but significant positive correlations with EDVI $(\mathrm{R}=0.28, P<0.01)$, ESVI $(\mathrm{R}=0.26, P<0.01)$, and SI $(\mathrm{R}=0.18, P=0.03)$. The logarithmic ARR also showed a weak positive correlation with EDVI $(\mathrm{R}=0.27, P<0.01)$, ESVI $(\mathrm{R}=0.26, P<0.01)$, and native $\mathrm{T} 1(\mathrm{R}=0.20, P=0.02)$ (Fig. 4$)$.

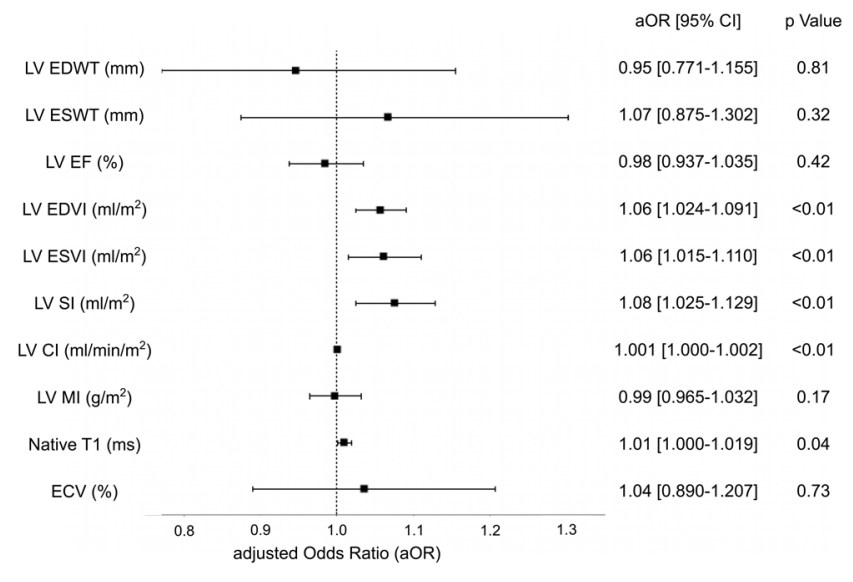

Figure 3

Association between MR parameters and subtypes of primary aldosteronism. Forest plot showing the adjusted odds ratio (aOR) and $95 \% \mathrm{Cl}$ of the aldosterone-producing adenoma group vs the bilateral hyperaldosteronism group (reference) for cardiac MR parameters. The aOR arises from the multivariable logistic regression analysis after controlling for age, sex, number of antihypertensive drugs, systolic and diastolic blood pressure, and disease duration. The horizontal lines show the $95 \% \mathrm{Cl}$ of the aOR for each parameter. $\mathrm{Cl}$, cardiac index; ECV, extra cellular volume; $E F$, ejection fraction; EDVI, end-diastolic volume index; EDWT, end-diastolic wall thickness; ESVI, end-systolic volume index; ESWT, end-systolic wall thickness; LV, left ventricle; MI, mass index; SI, systolic volume index. 


\section{Intra- and interobserver reproducibility}

The intraobserver ICCs of the native T1, postcontrast myocardial T1, and ECV measured in all patients were 0.90 (95\% CI: 0.87-0.93), 0.95 (0.85-0.96) and 0.86 (0.80-0.90), respectively. The interobserver ICCs of the native $\mathrm{T} 1$ values measured in all patients were 0.80 (95\% CI: 0.85-0.96), indicating good reliability.

\section{Discussion}

In this study, we demonstrated that APA, with higher hormonal activity than BHA, was associated with increased left ventricle EDVI, ESVI, SI, CI, and native $\mathrm{T} 1$ values on multivariable logistic regression analysis adjusted for age, sex, number of antihypertensive drugs, systolic and diastolic blood pressure, and disease duration. Furthermore, we revealed that these LV volumetric parameters and native $\mathrm{T} 1$ values had positive correlations with hormonal activity, namely PAC and ARR.

This is the first study evaluating LV volumetric parameters between the two groups by CMR, which provides a highly accurate and reproducible assessment of cardiac morphology and function. Hidaka et al. reported the differences in cardiac structure between APA and BHA using two-dimensional echocardiography (13). Their multiple linear regression analyses showed significantly larger left atrial volumes and left ventricular end-diastolic diameters in the APA than in the BHA group. Our results strongly support theirs in terms of the larger LV size in the APA group. PA patients demonstrate hyperkinetic circulation due to the increase of plasma volume, an inotropic effect of aldosterone, and/or enhanced sympathetic activity (24). Moreover, high aldosterone levels are associated with intravascular and intracardiac volume overloads (16). Therefore, our results indicate that APA patients with higher aldosterone activity promote more sodium and water retention, resulting in left ventricular dilatation and more hyperkinetic condition compared with the BHA group.

Our study showed no significant difference in LVMI between the two groups, in contrast to a previous echocardiographic study, which showed significantly increased LVM and higher prevalence of LVH in the APA group (13). The influence of aldosterone on LVM has been controversial. In a clinical study, Rossi et al. demonstrated that LVH was more pronounced in PA compared with EH due to increased deposition of collagen in the myocardial tissue (5), whereas Freel et al. demonstrated no significant differences in LVM or LVMI between the two groups in an MR study (16). This discrepancy may derive from the
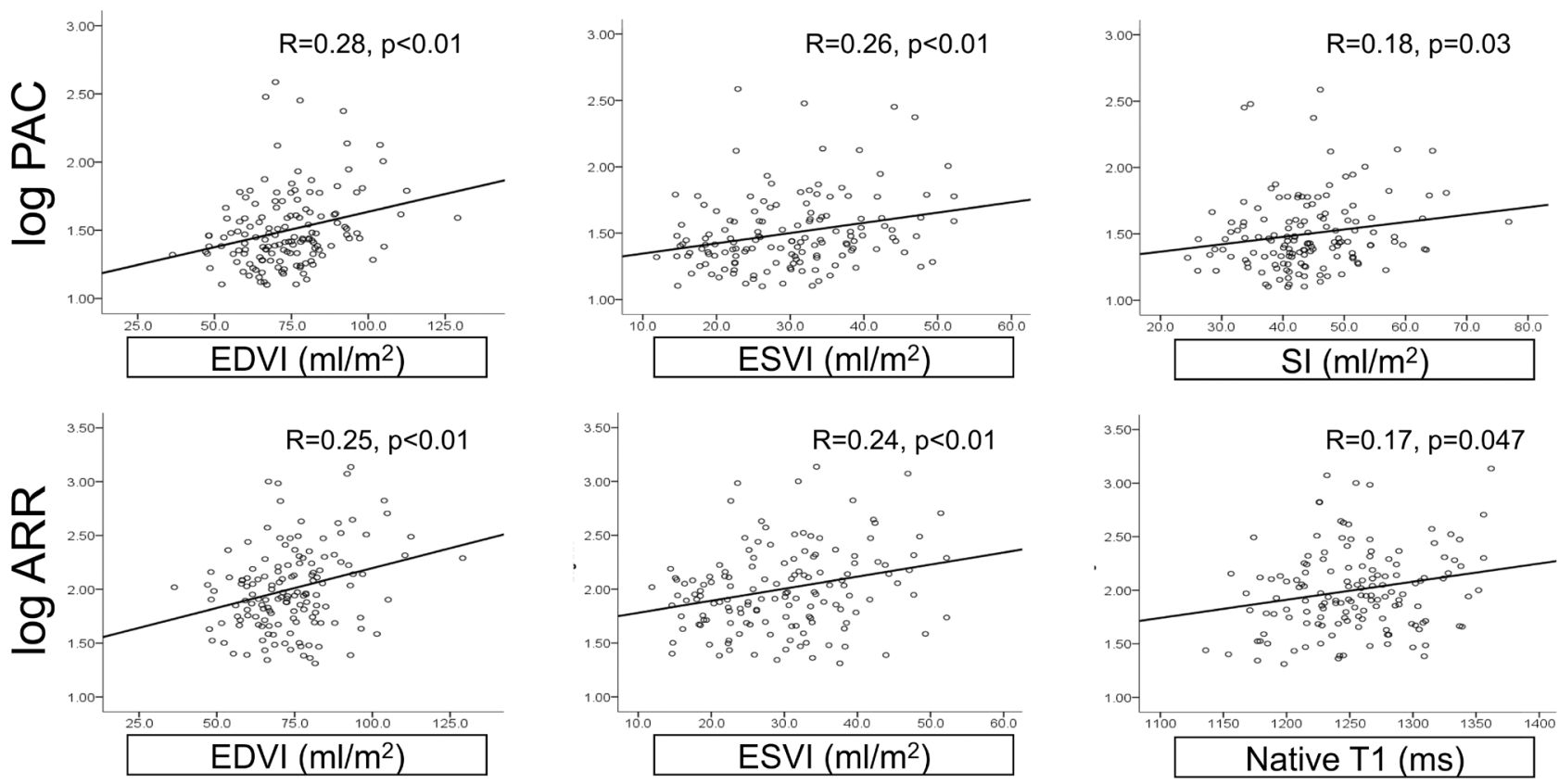

Figure 4

Correlation between MR parameters and hormonal data. Logarithmic transformation of plasma aldosterone concentration (PAC) and aldosterone to renin ratio (ARR) was performed to obtain normal distributions. The logarithmic PAC showed weak positive correlation with EDVI $(\mathrm{R}=0.28, P<0.01)$, ESVI $(\mathrm{R}=0.26, P<0.01)$, and $\mathrm{SI}(\mathrm{R}=0.18, P=0.03)$. The logarithmic ARR showed weak positive correlation with $\mathrm{EDVI}(\mathrm{R}=0.27, P<0.01), \mathrm{ESVI}(\mathrm{R}=0.26, P<0.01)$, and native T1 $(R=0.20, P=0.02)$. ARR, aldosterone to renin ratio; EDVI, end-diastolic volume index; ESVI, end-systolic volume index; SI, systolic volume index; PAC, plasma aldosterone concentration.

https://ec.bioscientifica.com https://doi.org/10.1530/EC-20-0504 (c) 2021 The authors Published by Bioscientifica Ltd

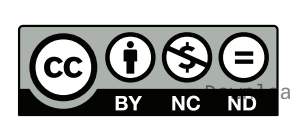

This work is licensed under a Creative Commons Attribution-NonCommercial-NoDerivatives 4.0 Internationabd ficense ifica . com at $04 / 26 / 2023 \quad 07: 24: 31 \mathrm{AM}$ 
different populations included in the studies. In a previous echocardiogram study by Hidaka et al., all APA patients had $\mathrm{LVH}$, defined as LVMI greater than $115 \mathrm{~g} / \mathrm{m}^{2}$ in men and $95 \mathrm{~g} / \mathrm{m}^{2}$ in women. However, in our study, both groups showed LVMI values within normal limits, reflecting relatively mild hypertension. The effect of aldosterone on LVH may depend on the degree of hypertension or disease duration more than on plasma aldosterone levels.

In our study, the presence of LGE reflecting myocardial fibrosis was not significantly different between the PA subtypes (16\% in APA vs $20 \%$ in BHA). The prevalence of LGE appears to be different from that reported in a previous MR study (70\% of PA patients and in 13\% of EH patients) (16). This discrepancy might derive from differences in the study populations, with our cohort characterized by relatively mild myocardial fibrosis. T1 mapping is a sensitive tool to detect and quantify diffuse and mild myocardial fibrosis or edema, but the normal value of native $\mathrm{T} 1$ varies depending on various parameters, including magnetic field strength, scan parameters, measurement method, age, and sex $(25,26,27)$. In our study, a larger proportion in the BHA group of female patients, whose normal value of native T1 is significantly higher than in males, did not result in a significant difference of native $\mathrm{T} 1$ between the two groups in univariable analysis; however, the difference became significant after controlling for demographic data in multivariable logistic regression analysis. Our results indicated that APA patients have mild myocardial fibrosis or edema, which could not be detected by LGE, more often than BHA patients. Past studies reported that T1 values played a role in discriminating cardiac events and prognosis in some cardiomyopathies $(28,29,30)$. Native T1 allowed the early detection of myocardial damage based on PA subtypes, which may become an important factor for cardiac risks in PA patients, however, the relationship between $\mathrm{T} 1$ values and cardiovascular events is still under investigation for patients with PA. Grytaas et al. reported that T1 mapping could not detect significant differences between PA and EH patients (18), but they did not take into account the subtypes of PA or demographic data that might have affected $\mathrm{T} 1$ values.

Moreover, our finding of a positive correlation between MR parameters and hormonal activity also supports the higher subclinical cardiac risk in APA patients and highlights the clinical importance of adrenal venous sampling (AVS) and surgical intervention in PA patients.

This study had several limitations. First, it is a retrospective, observational, single-center study, with the risk of selection bias. Second, follow-up MR studies are needed to reveal whether resection of the functioning adrenal tumors improves the MR findings in the APA group. Moreover, clinical follow-up is also needed to determine the long-term clinical significance of these results.

In conclusion, using a 3T MR scanner, we demonstrated that APA patients, characterized by higher hormonal activity than BHA patients, are associated with greater LV volumetric parameters and higher native T1 values, suggesting they have a higher risk of LV volume overload and mild myocardial damage. The results indicated the patients should be counseled about the potential for surgical intervention for unilateral disease after the diagnosis of PA. Larger sample sizes and follow-up studies are needed to determine whether these subclinical findings are related to clinical prognosis and whether early intervention can minimize the aldosterone-induced volume overload and myocardial injury.

\section{Supplementary materials}

This is linked to the online version of the paper at http://dx.doi.org/10.1530 /EC-20-0504.

\section{Declaration of interest}

The authors declare no conflicts of interest in this work that could be perceived as prejudicing the impartiality of the research reported.

\section{Funding}

This work was supported by JSPS KAKENHI Grant Number JP20H03613.

\section{Acknowledgements}

The authors would like to thank ASCl Cube (Advanced School for Core Investigators in Asian School of Cardiovascular Imaging of Asian Society of Cardiovascular Imaging) 2019 for helpful advice and comments, and Kyunghwa Han for statistical advice and comments. The authors would like to thank Dr Tatsuya Nishii for technical assistance with the experiments. The authors thank Yasuko Tsukada for helping to collect clinical data.

\section{References}

1 Omura M, Saito J, Yamaguchi K, Kakuta Y \& Nishikawa T. Prospective study on the prevalence of secondary hypertension among hypertensive patients visiting a general outpatient clinic in Japan. Hypertension Research 200427 193-202. (https://doi.org/10.1291/hypres.27.193)

2 Rossi GP, Bernini G, Caliumi C, Desideri G, Fabris B, Ferri C, Ganzaroli C, Giacchetti G, Letizia C, Maccario M, et al. A prospective study of the prevalence of primary aldosteronism in 1,125 hypertensive patients. Journal of the American College of Cardiology 200648 2293-2300. (https://doi.org/10.1016/j.jacc.2006.07.059)

3 Rossi GP, Sechi LA, Giacchetti G, Ronconi V, Strazzullo P \& Funder JW. Primary aldosteronism: cardiovascular, renal and metabolic implications. Trends in Endocrinology and Metabolism 2008 19 88-90. (https://doi.org/10.1016/j.tem.2008.01.006)

4 Sun Y, Zhang J, Lu L, Chen SS, Quinn MT \& Weber KT. Aldosteroneinduced inflammation in the rat heart: role of oxidative stress. American Journal of Pathology 2002161 1773-1781. (https://doi. org/10.1016/S0002-9440(10)64454-9) 
5 Rossi GP, Di Bello V, Ganzaroli C, Sacchetto A, Cesari M, Bertini A, Giorgi D, Scognamiglio R, Mariani M \& Pessina AC. Excess aldosterone is associated with alterations of myocardial texture in primary aldosteronism. Hypertension 200240 23-27. (https://doi. org/10.1161/01.hyp.0000023182.68420.eb)

6 Young M, Fullerton M, Dilley R \& Funder J. Mineralocorticoids, hypertension, and cardiac fibrosis. Journal of Clinical Investigation 199493 2578-2583. (https://doi.org/10.1172/JCI117269)

7 Farquharson CAJ \& Struthers AD. Aldosterone induces acute endothelial dysfunction in vivo in humans: evidence for an aldosterone-induced vasculopathy. Clinical Science 2002103 425-431. (https://doi.org/10.1042/cs1030425)

8 Delcayre C \& Silvestre JS. Aldosterone and the heart: towards a physiological function? Cardiovascular Research 199943 7-12. (https://doi.org/10.1016/S0008-6363(99)00088-7)

9 Monticone S, D'Ascenzo F, Moretti C, Williams TA, Veglio F, Gaita F \& Mulatero P. Cardiovascular events and target organ damage in primary aldosteronism compared with essential hypertension: a systematic review and meta-analysis. Lancet: Diabetes and Endocrinology 20186 41-50. (https://doi.org/10.1016/S2213-8587(17)30319-4)

10 Reincke M, Fischer E, Gerum S, Merkle K, Schulz S, Pallauf A, Quinkler M, Hanslik G, Lang K, Hahner S, et al. Observational study mortality in treated primary aldosteronism: the German Conn's registry. Hypertension 201260 618-624. (https://doi.org/10.1161/ HYPERTENSIONAHA.112.197111)

11 Lenders JWM, Eisenhofer G \& Reincke M. Subtyping of patients with primary aldosteronism: an update. Hormone and Metabolic Research 201749 922-928. (https://doi.org/10.1055/s-0043-122602)

12 Moors M, Williams TA, Deinum J, Eisenhofer G, Reincke M \& Lenders JWM. Steroid hormone production in patients with aldosterone producing adenomas. Hormone and Metabolic Research 201547 967-972. (https://doi.org/10.1055/s-0035-1565225)

13 Hidaka T, Shiwa T, Fujii Y, Nishioka K, Utsunomiya H, Ishibashi K, Mitsuba N, Dohi Y, Oda N, Noma K, et al. Impact of aldosteroneproducing adenoma on cardiac structures in echocardiography. Journal of Echocardiography 201311 123-129. (https://doi. org/10.1007/s12574-013-0168-y)

14 Haaf P, Garg P, Messroghli DR, Broadbent DA, Greenwood JP \& Plein S. Cardiac T1 mapping and extracellular volume (ECV) in clinical practice: a comprehensive review. Journal of Cardiovascular Magnetic Resonance 201618 89. (https://doi.org/10.1186/s12968 -016-0308-4)

15 Moon JC, Messroghli DR, Kellman P, Piechnik SK, Robson MD, Ugander M, Gatehouse PD, Arai AE, Friedrich MG, Neubauer S, et al. Myocardial T1 mapping and extracellular volume quantification: a Society for Cardiovascular Magnetic Resonance (SCMR) and CMR Working Group of the European Society of Cardiology consensus statement. Journal of Cardiovascular Magnetic Resonance 20131592. (https://doi.org/10.1186/1532-429X-15-92)

16 Freel EM, Mark PB, Weir RA, McQuarrie EP, Allan K, Dargie HJ, McClure JD, Jardine AG, Davies E \& Connell JM. Demonstration of blood pressure-independent noninfarct myocardial fibrosis in primary aldosteronism: a cardiac magnetic resonance imaging study. Circulation: Cardiovascular Imaging 20125 740-747. (https://doi. org/10.1161/CIRCIMAGING.112.974576)

17 Gaddam K, Corros C, Pimenta E, Ahmed M, Denney T, Aban I, Inusah S, Gupta H, Lloyd SG, Oparil S, et al. Rapid reversal of left ventricular hypertrophy and intracardiac volume overload in patients with resistant hypertension and hyperaldosteronism: a prospective clinical study. Hypertension 201055 1137-1142. (https:// doi.org/10.1161/HYPERTENSIONAHA.109.141531)
18 Grytaas MA, Sellevåg K, Thordarson HB, Husebye ES, Løvås K \& Larsen TH. Cardiac magnetic resonance imaging of myocardial mass and fibrosis in primary aldosteronism. Endocrine Connections 20187 413-424. (https://doi.org/10.1530/EC-18-0039)

19 Nishikawa T, Omura M, Satoh F, Shibata H, Takahashi K, Tamura N, Tanabe A \& Task Force Committee on Primary Aldosteronism, The Japan Endocrine Society. Guidelines for the diagnosis and treatment of primary aldosteronism - The Japan Endocrine Society 2009. Endocrine Journal 201158 711-721. (https://doi.org/10.1507/endocrj.EJ11-0133)

20 Hundley WG, Bluemke D, Bogaert JG, Friedrich MG, Higgins CB, Lawson MA, McConnell MV, Raman SV, Rossum AC, Flamm S, et al. Society for Cardiovascular Magnetic Resonance guidelines for reporting cardiovascular magnetic resonance examinations. Journal of Cardiovascular Magnetic Resonance 200911 5. (https://doi. org/10.1186/1532-429X-11-5)

21 Satani N, Ota H, Seiji K, Morimoto R, Kudo M, Iwakura Y, Ono Y, Nezu M, Omata K, Ito S, et al. Intra-adrenal aldosterone secretion: segmental adrenal venous sampling for localization. Radiology 2016 278 265-274. (https://doi.org/10.1148/radiol.2015142159)

22 Rossi GP, Auchus RJ, Brown M, Lenders JWM, Naruse M, Plouin PF, Satoh F \& Young Jr WF. An expert consensus statement on use of adrenal vein sampling for the subtyping of primary aldosteronism. Hypertension 201463 151-160. (https://doi.org/10.1161/ HYPERTENSIONAHA.113.02097)

23 Satoh F, Abe T, Tanemoto M, Nakamura M, Abe M, Uruno A, Morimoto R, Sato A, Takase K, Ishidoya S, et al. Localization of aldosterone-producing adrenocortical adenomas: significance of adrenal venous sampling. Hypertension Research 200730 1083-1095. (https://doi.org/10.1291/hypres.30.1083)

24 Tarazi RC, Ibrahim MM, Bravo EL \& Dustan HP. Hemodynamic characteristics of primary aldosteronism. New England Journal of Medicine 1973289 1330-1335. (https://doi.org/10.1056/NEJM197312202892502)

25 Kellman P \& Hansen MS. T1-mapping in the heart: accuracy and precision. Journal of Cardiovascular Magnetic Resonance 2014162. (https://doi.org/10.1186/1532-429X-16-2)

26 Dabir D, Child N, Kalra A, Rogers T, Gebker R, Jabbour A, Plein S, Yu CY, Otton J, Kidambi A, et al. Reference values for healthy human myocardium using a T1 mapping methodology: results from the International T1 Multicenter Cardiovascular Magnetic Resonance Study. Journal of Cardiovascular Magnetic Resonance 20141669. (https://doi.org/10.1186/s12968-014-0069-x)

27 Rauhalammi SMO, Mangion K, Barrientos PH, Carrick DJA, Clerfond G, McClure J, McComb C, Radjenovic A \& Berry C. Native myocardial longitudinal (T1) relaxation time: regional, age, and sex associations in the healthy adult heart. Journal of Magnetic Resonance Imaging 201644 541-548. (https://doi.org/10.1002/ jmri.25217)

28 Puntmann VO, Carr-White G, Jabbour A, Yu CY, Gebker R, Kelle S, Hinojar R, Doltra A, Varma N, Child N, et al. T1-mapping and outcome in nonischemic cardiomyopathy: all-cause mortality and heart failure. JACC: Cardiovascular Imaging 20169 40-50. (https://doi. org/10.1016/j.jcmg.2015.12.001)

29 Xu J, Zhuang B, Sirajuddin A, Li S, Huang J, Yin G, Song L, Jiang Y, Zhao S \& Lu M. MRI T1 mapping in hypertrophic cardiomyopathy: evaluation in patients without late gadolinium enhancement and hemodynamic obstruction. Radiology 2020294 275-286. (https:// doi.org/10.1148/radiol.2019190651)

30 Banypersad SM, Fontana M, Maestrini V, Sado DM, Captur G, Petrie A, Piechnik SK, Whelan CJ, Herrey AS, Gillmoreet JD, et al. T1 mapping and survival in systemic light-chain amyloidosis. European Heart Journal 201536 244-251. (https://doi.org/10.1093/eurheartj/ehu444)

Received in final form 30 October 2020

Accepted 25 November 2020

Accepted Manuscript published online 27 November 2020 https://ec.bioscientifica.com https://doi.org/10.1530/EC-20-0504 (c) 2021 The authors Published by Bioscientifica Ltd
This work is licensed under a Creative Commons Attribution-NonCommercial-NoDerivatives 4.0 International License.ifica com at $04 / 26 / 2023 \quad 07: 24: 31$ M 